\title{
Eventos en la industria de los videojuegos. El caso Fortnite
}

\author{
Bideo-jokoen industriako ekitaldiak. Fortnite kasua \\ The use of the events in the videogame industry: The Fortnite case
}

\author{
Antonio Raúl Fernández Rincón`, Pedro Antonio Hellín Ortuño, \\ Antonio Castillo Esparcia \\ Universidad de Murcia, Universidad de Málaga
}

RESUMEN: Los videojuegos constituyen hoy una de las industrias creativas y culturales más relevantes. Los desarrolladores producen estrategias de comunicación transmedia para que sus públicos interaccionen socialmente desde la perspectiva informativa, opinativa y narrativa. El objetivo es mantener el interés y la expectación hacia el videojuego, estableciendo relaciones de adaptación e integración entre la organización y sus públicos. En este artículo estudiamos las características del videojuego Fortnite, las dinámicas promocionales que se generan y los medios empleados. Asistimos a un claro ejemplo de transmedia branding donde los individuos co-participan, co-crean, se apropian y recirculan informaciones y experiencias, estableciendo una narrativa común, de tipo afectivo, que integra a todos esos canales comunicativos formando una interacción y experiencia alrededor del videojuego.

PALABRAS CLAVE: Transmedia; videojuegos; Fortnite; comunicación; relaciones públicas; eventos.

ABSTRACT: Videogames nowadays constitute one of the most relevant creative and cultural industries. The developers produce transmedia communication strategies so that their audiences interact socially from the informational, opinion and narrative perspective. The goal is to maintain interest and expectation towards the videogame, building relationships of adaptation and integration between the organization and its audiences. In this article we study the characteristics of Fortnite, the promotional dynamics that are generated and the media used. We are witnessing a clear example of transmedia branding where the players co-participate, co-create, appropriate and recirculate information and experiences, establishing a common narrative, in an emotional way, that integrates all these communication channels, forming an interaction and experience around the videogame.

KEYWORDS: Transmedia, videogames; Fornite; communication; public relations; events.

\footnotetext{
* Correspondencia a / Corresponding author: Antonio Raúl Fernández Rincón. Facultad de Comunicación y Documentación de la Universidad de Murcia. C. Campus Universitario, 8 (30100 Murcia) - antonioraul.fernandez@um.es - https://orcid.org/0000-0001-7143-2175

Cómo citar / How to cite: Fernández Rincón, Antonio Raúl; Hellín Ortuño, Pedro Antonio; Castillo Esparcia, Antonio (2021). «Eventos en la industria de los videojuegos. El caso Fortniten, Zer, 26(51), 35-53. (https://doi.org/10.1387/zer.23062).

Recibido: 17 septiembre, 2021; aceptado: 7 noviembre, 2021.

ISSN 1137-1102 - eISSN 1989-631X / (c) 2021 UPV/EHU

(c) (1) $(\Theta)$ Esta obra está bajo una Licencia

cro ${ }_{\mathrm{BC}}$ Creative Commons Atribución-NoComercial-SinDerivadas 4.0 Internacional
} 


\section{Introducción}

La industria del videojuego en España es un sector de pequeño tamaño en el presente, pero con una gran potencialidad de futuro. En 2016 la Asociación Española de Videojuegos (AEVI, 2018) ya cifraba la producción generada por el sector en 1.177 millones de euros, lo que representaba el 0,1\% del PIB. Además, por el efecto arrastre que la actividad del sector tiene sobre el resto de ramas, a través de sus compras a proveedores, se estimó su impacto económico total en 3.577 millones de euros. El impacto total del sector del videojuego era de 3.577 millones de euros y 22.828 empleos. En 2018 el consumo de videojuegos ya lideraba las industrias culturales y creativas en España, situándose en el puesto noveno a nivel mundial.

Los desarrolladores y comercializadores de los videojuegos configuran un entramado transmedia (Scolari, 2009: 600) de redes sociales, aplicaciones móviles, canales de video, wikies, galerías fotográficas, blogs y microsites web que albergan la simbología, los relatos y el discurso de los productos, gestionando las relaciones con sus públicos. Estos contenidos son replicados a su vez, en múltiples canales informativos propios o ajenos, en medios especializados o como parte integrante de la información sobre tecnología y/o entretenimiento de los medios tradicionales. La propia dinámica productiva de los videojuegos, inmersa en el actual escenario tecno-comunicativo, propicia un flujo constante de actualizaciones y novedades que trabaja para mantener el interés de los públicos, alargando la vida útil de los productos. La ausencia de soportes fisicos como el $c d$-rom o anteriormente, las cintas analógicas, hacen del videojuego un producto abierto, flexible, sujeto a cambios y susceptible de evolucionar según las necesidades de sus creadores o las expectativas de sus públicos. En este entorno, cada lanzamiento, actualización o novedad en el juego, se gestiona en términos de comunicación como un evento destinado a renovar el interés de los públicos hacia el juego. Es aquí donde se encuentra el objeto de nuestro estudio, los eventos como herramientas estratégicas de relación con los públicos en el sector de los videojuegos. Para el estudio de estos eventos o acontecimientos especiales nos hemos centrado en Fortnite, un videojuego lanzado en 2017 por la compañía estadounidense Epic Games. Las razones que justifican su elección para este estudio son dos: por una parte, se trata de un videojuego que emplea de forma extensiva los eventos para su promoción y, por otra parte, estamos ante uno de los videojuegos más exitosos de los últimos tiempos, tal y como demuestran las fuentes consultadas (tabla 1).

Los videojuegos han aumentado de manera ostensible su presencia en numerosos ámbitos sociales, económicos, educativos y comunicativos. Sin embargo, no debe olvidarse que los videojuegos están gestionados por empresas y, por ende, deben contar y cuentan con estrategias de comunicación y de marketing. En esta investigación, se analiza el papel de las estrategias de comunicación de los videojuegos desde la perspectiva de las relaciones con los públicos (relaciones públicas). De manera concreta, nos centramos en cómo se gestionan los públicos de estos productos 
lo que implica planificar acciones y herramientas de comunicación que se acomoden a las características de ese tipo de público.

TABLA 1

Logros y reconocimientos de Fornite

\begin{tabular}{lll}
\hline \multicolumn{1}{c}{ Logros } & \multicolumn{1}{c}{ Fuente } & Año \\
\hline Juego más descargado. Modalidad free-to-play & PlayStation Store & 2018 \\
Juego más descargado & E-shop Switch & 2018 \\
Tráiler más visto: «Season 5» & gamesindustry.biz & 2018 \\
Top 1 juegos en Twitter & gamesindustry.biz & 2018 \\
Top 1 juegos con más cobertura de noticias & gamesindustry.biz & 2018 \\
Top 3 en TwitchTv & twitchMetrics.net & 2018 \\
Mejor juego competitivo multijugador & Webby Awards & 2018 \\
Mejor juego en curso & Game Critics Awards & 2018 \\
Juego de elección & Teen Choice Awards & 2018 \\
Top 9 videojuegos más jugados del mundo & Epic Games & 2019 \\
Top 1 descargas. AppStore España & appannie.com & 2019 \\
Top 7 en juegos de PC & superdataresearch.com & 2019 \\
Top 3 descargas. AppStore España & appannie.com & 2019 \\
Nominado «Best Evolving Game» Bafta Games Award & Imbd.com & 2020 \\
\hline
\end{tabular}

Fuente: elaboración propia.

\subsection{El eVEnto COMO tÉCNiCA DE RELACIONES PÚBLICAS}

Los eventos son una técnica recurrente en relaciones públicas que no cuentan con una definición consensuada a día de hoy. Tal y como afirma Bowdin (2006: 13) tan solo hay un cierto acuerdo limitado en términos estandarizados y categorías de uso. Entre las definiciones más usadas por la literatura sobre eventos se encuentran las de Donald Getz o Joe Goldblatt. Para Getz (2012: 18) un evento es: «Un suceso en un lugar y momento determinados; un conjunto especial de circunstancias; de ocurrencia notable» es decir, un fenómeno temporal con unos objetivos, un principio y un final planificado. Goldblatt (1990) incluye además, el factor ceremonial y ritualístico como algo propio de estos actos. Ambos autores coinciden en señalar la importancia del concepto de planificación para distinguir los eventos espontáneos de los eventos planificados, encaminados a alcanzar metas específicas a través de la experiencia de los participantes. Solo los eventos planificados podrían formar parte del campo académico que Getz (2012) denomina Event Studies y que vendría a dar respuesta al desarrollo de la industria de los eventos en los últimos años. La planificación estratégica de los eventos como herramienta de relaciones públicas ha experimentado un importante crecimiento a partir de la década de los noventa del siglo xx, del siglo xx (Xifra, 2007; Allen, 2008; Yeoman et al., 2011; Da Câmara 
Correia, 2017). En términos generales, un evento es un acontecimiento utilizado para generar la atención de los públicos de una organización.

En esta línea podemos clasificar los eventos en torno a tres categorías: eventos naturales, eventos artificiales directos y pseudoeventos. Los eventos naturales son aquellos que se manifiestan espontáneamente en la vida de una organización y que no requieren intervención por parte del departamento de relaciones públicas. Los eventos artificiales directos son aquellos que pudiéndose dar de forma espontánea requieren una intervención del profesional de relaciones públicas con vistas a asegurar la eficacia en el cumplimiento de los objetivos propuestos. Los pseudoeventos o también denominados pseudo-acontecimientos (Xifra, 2003: 224) se centran en conseguir publicity, donde la comunicación no es suscrita por la organización, sino por el medio de comunicación, que es quien controla el mensaje para lograr la repercusión deseada. La creación de eventos y pseudoeventos no es reciente. Arceo Vacas (1988) sitúa su origen en la década de 1920 y a raíz de las actividades del empresario Phineas T. Barnum, para obtener presencia en los medios. Este momento coincide con el desarrollo inicial de la denominada publicity y los inicios de las relaciones públicas modernas. Hoy se considera una información sobre actividades, actos o eventos de una organización que es generada, difundida y controlada por un medio de comunicación a instancias de aquella (Xifra, 2010). El pseudoevento sitúa a las relaciones públicas en el espacio más próximo a la espectacularidad y la artificialidad de los vínculos entre las organizaciones y sus públicos.

El ecosistema digital generó un nuevo conglomerado de medios y sinergias corporativas (Edwards, 2012) que ofrece nuevas oportunidades y canales para una comunicación basada en la integración y la readaptación de los mensajes (Nicolás Ojeda et al., 2019). Todo ello ha venido a enriquecer el esquema clásico de Shannon y Weaver, adaptado después por Jakobson. Los fundamentos de este modelo se mantienen intactos pero las condiciones de emisión y recepción han mutado y ahora son intercambiables (Aced, 2013). El interés por la comunicación masiva ha dado paso a una comunicación más segmentada y en red que Castells (2009) ya definió como "autocomunicación de masas», donde los límites para la circulación de los mensajes se han diluido y el emisor puede alcanzar audiencias globales gracias a los nuevos medios. La era de la inteligencia colectiva ha dado lugar a un nuevo y potente «nosotros» que es mucho más inteligente que cualquier "yo» singular (Libert y Spector, 2008). Para Castillo Esparcia y Almansa (2005: 139) las tecnologías de la comunicación desempeñan un papel remarcable en las estrategias externas de las organizaciones porque coadyuvan a que la comunicación que realicemos llegue más rápidamente y de mejor manera a nuestros interlocutores.

En la gestión estratégica de la comunicación en las organizaciones los medios sociales han generado salas de prensa virtuales, publicity, blogs o eventos 2.0 que se benefician de estos en su organización, realización, comunicación y difusión (Cam- 
pillo et al., 2014: 58). Los objetivos de estos eventos están relacionados con la generación de notoriedad, engagement y afluencia virtual o física.

El diseño de eventos 2.0 pasa por habilitar espacios online donde favorecer la participación activa y la interacción de los asistentes sin olvidar la fuerza de la presencialidad y su arraigada tradición (Estanyol I Casals, 2012). Para Rodríguez (2011) lo verdaderamente avanzado e innovador de este tipo de eventos reside en el cambio de paradigma que supone dirigirse a aforos ilimitados y a no tener dependencia de los medios de comunicación tradicionales. Los espectadores pueden seguirlos a través de la red, en directo o transcurrido un tiempo, a través de YouTube, Facebook o cualquier blog especializado. La tecnología supone para los eventos una mayor implicación del público en todas sus fases; al principio para fomentar el deseo de asistir o presenciarlo, después para implicarlo en su funcionamiento y, por último, para evaluar su éxito y áreas de mejora (Rodríguez, 2014).

En el contexto de la teoría sobre el transmedia, las organizaciones han desarrollado numerosas estrategias para poder establecer vínculos satisfactorios con sus públicos. Para ello, es esencial, una gestión adecuada de estos, con el despliegue investigador que nos permita conocer cómo son, cómo se informan, qué medios utilizan o a qué soportes recurren para sus ámbitos informacionales, opinativos, sociales o lúdicos. Los jugadores / fans / consumidores no cumplen un mero rol receptor sino que modifican, difunde y expanden los argumentos de los textos que dan sentido a diferentes narrativas individuales, propias de cada producto (San Nicolás Romera et al., 2015: 115). En este sentido, el sector de los videojuegos posee unas características específicas como consecuencia del perfil socioeconómico de sus usuarios que entroncan con el sector juvenil. La diversidad de plataformas, espacios o soportes comunicativos con los que interactúan aportan complejidad, pero al mismo tiempo multiplican las posibilidades de establecer vínculos con esos públicos.

Desde esta perspectiva de la transmediación, Tenderich (2014: 16) ha definido al transmedia branding como: «Un proceso de comunicación en el que la información sobre una marca se empaqueta en una narrativa integrada, que se dispersa en contribuciones únicas a través de múltiples canales, con el propósito de crear una experiencia de marca interactiva y atractiva».

Para poder establecer los aspectos más relevantes de la perspectiva transmedia branding es ineludible apelar a la interactividad de las plataformas y al empoderamiento de los públicos para generar acciones de vínculos recíprocos con las organizaciones, lo que, sin duda, imbrica una relación de tipo emocional que puede generar comunidades de públicos alrededor de las organizaciones y de sus valores (Scolari et al., 2019; Campillo Alhama y Martínez Sala, 2019). El diseño de Fortnite empuja a los jugadores fuera de la plataforma del juego a sitios como YouTube y Twitch para aprender habilidades sobre cómo jugar, cómo completar diferentes desafios diarios y semanales, para actualizarse 
sobre eventos y cambios en el juego, lo que sugiere una conceptualización más amplia de lo que significa «jugar» a Fortnite (Carter et al., 2020). En este sentido, Aarsand (2013: 124), habla de culturas de juego como culturas participativas que implican mucho más que jugar. Más allá de la interacción práctica del jugador con el juego, existe un compromiso más amplio con él, desde ver jugadores expertos en Twitch hasta realizar bailes en la escuela. Videojuegos como Fortnite, por tanto, están conectados con una ecología de medios digitales emergentes que influyen en la vida de los jugadores.

\subsection{Antecedentes y Objetivos de LA InVESTigación}

A la clasificación de estudios sobre videojuegos aportada hace más de una década y que contemplaba tres áreas principales de estudio: videojuegos como narraciones, videojuegos como juegos y videojuegos como producto cultural (Gómez García, 2007: 71), pueden sumarse otros enfoques como: videojuegos como herramientas; estudios sobre hábitos de consumo y perfiles de videojugadores. En nuestro estudio, el interés se centra en las acciones generadas por los videojuegos desde el marco de la comunicación organizacional y las relaciones públicas. El videojuego Fortnite es un bien de consumo y un fenómeno social, al mismo tiempo que podría revelar interesantes dinámicas de comunicación e interacción con los públicos y que abre un campo de acción para la disciplina.

Los objetivos definidos para este estudio son:

1. A modo de contextualización, explorar las características del videojuego y las dinámicas promocionales que la compañía propietaria ha realizado desde su lanzamiento.

2. Describir los eventos realizados y los medios empleados para su difusión. El objetivo es lograr una categorización de los mismos para así poder extraer conclusiones sobre sus características y peculiaridades.

3. Detectar rasgos en común que mantienen estas iniciativas con las definiciones de evento, evento 2.0 y pseudoevento como técnicas de relaciones públicas. Intentamos generar una reflexión y/o discusión en nuestra disciplina en torno a la industria cultural de los videojuegos y su potencialidad como campo de trabajo y estudio.

\section{Metodología}

El método empleado en esta investigación es el estudio de caso en profundidad (case study) que se fundamenta en una etnografia comunicativa y que busca obtener información de carácter cualitativo sobre el fenómeno estudiado. La finalidad del estudio de caso es descriptiva y explicativa. A partir de la clasificación de Yin (1994), 
optamos por el formato "caso simple, diseño incrustado", porque el estudio se desarrolla sobre un solo objeto, proceso o acontecimiento, usando dos o más unidades (Díaz De Salas et al., 2011). El estudio cualitativo que presentamos aquí es de tipo longitudinal y retrospectivo (Rodríguez Gómez et al., 1996). La medición se realizó a lo largo de un periodo de tiempo pasado, concretamente entre septiembre de 2018 y agosto de 2021. Este periodo coincide con la fase posterior a su lanzamiento en 2017 y el inicio de una política de eventos destinados a renovar de forma continuada el interés por el juego.

Debido a la escasez de fuentes oficiales que aporten datos objetivos sobre diferentes aspectos del sector como la distribución, las ventas, las descargas, los jugadores y otros, hemos optado por generar un conglomerado de agentes que intervienen en el sector y, a partir de ahí, poder tener una perspectiva explorativa del fenómeno, reduciendo el posible sesgo de los propios distribuidores (tabla 2).

TABLA 2

Fuentes consultadas

\begin{tabular}{|c|c|c|c|}
\hline Fuente & Categoría & Sección & Link \\
\hline Marca & Diario online & Fortnite & https://bit.ly/2ks5J5W \\
\hline Grupo Prisa (El País + Diario As) & $\begin{array}{l}\text { Diario online } \\
\text { Diario online }\end{array}$ & $\begin{array}{l}\text { Fortnite } \\
\text { Meristation }\end{array}$ & $\begin{array}{l}\text { https://bit.ly/2kNSzR9 } \\
\text { https://bit.ly/2kxjCjq }\end{array}$ \\
\hline La Vanguardia & Diario online & Vangamers & https://bit.ly/2kxj9Oc \\
\hline El Mundo & Diario online & Videojuegos & https://bit.ly/2m76sKc \\
\hline$A B C$ & Diario online & Videojuegos & https://bit.ly/2kZPjSH \\
\hline El Periódico & Diario online & Videojuegos & https://bit.ly/2m1RfKy \\
\hline El Mundo Deportivo & Diario online & Videojuegos & https://bit.ly/2munItf \\
\hline La Razón & Diario online & Videojuegos & https://bit.ly/2kZ0Ilw \\
\hline Público & Diario online & Videojuegos & https://bit.ly/2kpMrhw \\
\hline El Plural & Diario online & Gaming & https://bit.ly/2kueFb7 \\
\hline El Español & Diario online & Vandal & https://bit.ly/2CZexHL \\
\hline Okdiario & Diario online & Videojuegos & https://bit.ly/2mtmXAD \\
\hline DotEsports & $\mathrm{Blog}$ & Fortnite & https://dotesports.com \\
\hline Hypebeast & $\mathrm{B} \log$ & Gaming & https://bit.ly/2ATgfbb \\
\hline SuperData Research & Consultora & - & https://bit.ly/1K172sj \\
\hline AppAnnie & Consultora & - & https://bit.ly/1F1kN8r \\
\hline Gamesindustry & Consultora & - & https://bit.ly/2L7GDp0 \\
\hline Statista & Consultora & - & https://es.statista.com \\
\hline$A E V I$ & Asociación & - & http://www.aevi.org.es \\
\hline TwitchMetrics & Métricas Twitch Tv & - & https://bit.ly/2ns4vcm \\
\hline Semrush & Métricas Web & - & https://bit.ly/31pnX7U \\
\hline Metricool & Métricas RR.SS. & & https://bit.ly/31w9uqH \\
\hline Switch e-shop & Distribuidor & - & https://bit.ly/2VwjCOO \\
\hline PlayStation Store & Distribuidor & - & https://bit.ly/2OxTEsI \\
\hline
\end{tabular}

Fuente: elaboración propia. 
Hemos realizado un seguimiento de las plataformas digitales del propio juego (tabla 3). Se indica la cuantificación de la audiencia según las propias plataformas y en términos de seguidores (YouTube, Instagram, Twitter) y visualizaciones totales (TwitchTv). Para la web Epic Games se aportan datos sobre el tráfico orgánico en España, según la plataforma SemRush (https://www.semrush.com).

TABLA 3

Plataformas digitales del juego

\begin{tabular}{lrrrrl}
\hline Plataforma & $\begin{array}{r}\text { Audiencia } \\
(09 / 2018)\end{array}$ & \multicolumn{1}{c}{$\begin{array}{c}\text { Audiencia } \\
(09 / 2019)\end{array}$} & $\begin{array}{r}\text { Audiencia } \\
(04 / 2020)\end{array}$ & $\begin{array}{r}\text { Audiencia } \\
(08 / 2021)\end{array}$ & \multicolumn{1}{c}{ Link } \\
\hline Epic Games & 910.000 & 2.743 .072 & 1.993 .302 & 847.700 & https://bit.ly/2UveE3V \\
Youtube & 6.760 .000 & 7.360 .000 & 8.510 .000 & 10.700 .000 & $\begin{array}{l}\text { https://bit.ly/2wnNu6T } \\
\text { Instagram }\end{array}$ \\
TwitchTv & 53.450 .000 & 22.300 .000 & 22.700 .000 & 26.500 .000 & fortnite \\
Twitter & 8.380 .000 & 9.500 .000 & 10.100 .000 & 12.800 .000 & @FortniteGame \\
\hline
\end{tabular}

Fuente: elaboración propia.

\section{Resultados. El fenómeno Fortnite}

Fortnite es un videojuego del género battle royale (supervivencia) que presenta varios modos gestionados desde un mismo motor y con reglas similares. Es gratuito y admite micro-pagos. Una vez que el jugador lo ha descargado, para concluir los retos o conseguir ventaja sobre otros jugadores puede efectuar compras internas a través de la moneda virtual $(v$-buck). Numerosos analistas de videojuegos e investigadores han analizado el éxito de un título que, solo en el primer año de su lanzamiento ganó más de mil millones de dólares (Parker, 2018; Chan, 2018; Palmeri y Pendleton, 2018; Flanagan, 2018; Tassi, 2018; Parkin, 2018; Marlatt, 2019). Es un juego de operaciones militares en el que el jugador es lanzado en paracaídas sobre un mundo hostil junto con otros 99 jugadores armados con solo un pico. Los jugadores usan su herramienta para construir refugios, reunir materiales y recolectar armas para sobrevivir. Su éxito se puede atribuir a factores como la accesibilidad, la escasa dificultad del aprendizaje, su informalidad, el colorido, las actualizaciones constantes y la oferta continuada de complementos cosméticos (Montes, 2018). El videojuego está disponible en la mayoría de plataformas y sistemas operativos (Microsoft Windows, MacOS, Xbox One, iOS, Android, PlayStation 4, Nintendo Switch). Según la propia compañía creadora del juego, Fornite contaba a finales de 2019 con 250 millones de jugadores registrados y 10.8 millones de jugadores concurrentes.

La renovación del interés hacia el juego se genera a partir de actualizaciones periódicas (temporadas) y eventos que coinciden con las estaciones del año. Estos 
acontecimientos se notifican en el propio juego, en los canales del juego (YouTube; TwicthTv; Instagram; Twitter y Facebook) y en los medios digitales de información. En el periodo estudiado encontramos 20 eventos que después hemos agrupado en 7 categorías (tabla 4).

TABLA 4

Eventos por orden cronológico

\begin{tabular}{|c|c|c|c|}
\hline Evento & Fecha & Evento & Fecha \\
\hline Drake y Ninja & $09 / 2018$ & Aquaman & $7 / 2020$ \\
\hline Pantera Negra & $09 / 2018$ & Capitán América & $7 / 2020$ \\
\hline Día de los Muertos en México & $10 / 2018$ & Thor y Galactus & $8 / 2020$ \\
\hline Burger King & $11 / 2018$ & Concierto Anderson Paak & $09 / 2020$ \\
\hline Australia Open Summer Smash & $01 / 2019$ & Concierto Dominique Fike & $09 / 2020$ \\
\hline Fortnite World Cup 2019 & $02 / 2019$ & Concierto J Balvin & $10 / 2020$ \\
\hline Concierto DJ Marshmello & $02 / 2019$ & Copa Daredevil & $10 / 2020$ \\
\hline John Wick & $04 / 2019$ & Evento BTS & $10 / 2020$ \\
\hline Los Vengadores (Endgame) & $04 / 2019$ & Concierto LILNasX & $11 / 2020$ \\
\hline Air Jordan & $05 / 2019$ & Galactus & $12 / 2020$ \\
\hline Stranger Things & $07 / 2019$ & Mandalorian & $12 / 2020$ \\
\hline Borderlands & $08 / 2019$ & Black Panther & $12 / 2020$ \\
\hline 80 cumpleaños Batman & $09 / 2019$ & Torneo TheGrefg & $01 / 2021$ \\
\hline La caída del meteorito & $09 / 2019$ & Noche de Cortos & $02 / 2021$ \\
\hline Star Wars & $12 / 2019$ & San Valentín & $02 / 2021$ \\
\hline Festival de Invierno-Navidad & $12 / 2019$ & Copa Flash DC & $02 / 2021$ \\
\hline Australia Open Summer Smash & $02 / 2020$ & Copa Neymar JR & $03 / 2021$ \\
\hline Copa de Celebración & $02 / 2020$ & Concierto Kaskade & $03 / 2021$ \\
\hline Concierto Travis Scott & $04 / 2020$ & Concurso de fotografia furtiva & $03 / 2021$ \\
\hline Concurso dibujo magistral & $05 / 2020$ & Copa Fuga Primaveral y Pascua & $04 / 2021$ \\
\hline Películas Christofer Nolan & $06 / 2020$ & Fortnite X Havaianas & $04 / 2021$ \\
\hline DillonFrancis, S-Aoki; Deadmau5 & $06 / 2020$ & Fortnite X NBA & $05 / 2021$ \\
\hline Diplo; Major Lazer; Noah Cyrus & $06 / 2020$ & Copa Uefa EURO 2020 & $06 / 2021$ \\
\hline El Dispositivo & $06 / 2020$ & Concierto EasyLife & $06 / 2021$ \\
\hline Evento Diplo & $07 / 2020$ & Ídolos Harry Kane y Marco Reus & $06 / 2021$ \\
\hline Tertulias raciales y políticas & $07 / 2020$ & Ídolos Bugha & $07 / 2021$ \\
\hline Fortnite vs Apple & $08 / 2020$ & Fortnite X Ferrari 296 GTB & $07 / 2021$ \\
\hline Evento Llama-Rama & $09 / 2020$ & Ídolos LeBron James & $07 / 2021$ \\
\hline Supercopa $1 \mathrm{M}$ de dólares & $11 / 2020$ & Concierto Ariana Grande & $08 / 2021$ \\
\hline
\end{tabular}

Fuente: elaboración propia.

\subsection{Eventos Relacionados CON PROducciones Cinematográficas}

Fornite es uno de los más importantes frentes comerciales del gigante del entretenimiento The Walt Disney Company. Los creadores de Mickey Mouse son hoy 
el mayor conglomerado de medios de comunicación y entretenimiento que engloba diferentes marcas como: 21st Century Fox; Walt Disney Pictures; Pixar Animation Studios; Lucasfilm; Marvel Studios; Disney+; ESPN+; ABC Entertainment; Hulu; The History Channel y otras (https://thewaltdisneycompany.com). Se han realizado acciones a propósito de la película Pantera Negra (el primer superhéroe negro de la historia). También se han incluido a Star-Lord (personaje de la película Guardianes de la Galaxia) y referencias explícitas a la saga de Los Vengadores de 2019 (The End Game). En abril de 2019 se produjo el evento John Wick, una saga de películas de acción con influencias del cine oriental de artes marciales. La serie de ciencia ficción Stranger Things de Netflix también ha sido insertada en Fortnite. El evento que unía ambos mundos fue anunciado en julio de 2019 con motivo del estreno de su tercera temporada. Con la celebración en 2019 del 80 aniversario del nacimiento del popular superhéroe Batman, Epic Games anunció en septiembre de 2019 la inclusión de los personajes, sus equipamientos y la propia ciudad de Gotham dentro de Fortnite. La colaboración fue anunciada con el correspondiente tráiler en la cuenta oficial del juego en YouTube. En diciembre de 2019 y antes de la celebración de los premios Game Awards, Fornite anunciaba el evento de la película Star Wars: El ascenso de Skywalker. Además de insertar personajes y objetos para su compra dentro del juego, el 14 de diciembre se emitió un spot con varias escenas inéditas de la película. A finales de 2020 se desarrolló un evento relacionado con la serie Mandalorian, asociada a la saga de Star Wars y que se emitió en el canal temático Disney+.

\subsection{Hostelería, OCiO y DEPORTES}

En noviembre de 2018 la compañía Burger King se unió a Fortnite para el evento "Guerra de comida», donde los jugadores se dividían en dos equipos, unos defendiendo a las hamburguesas y otros a las pizzas. Este reto fue empleado para lanzar una campaña junto al youtuber TheGrefg. Burger King tuvo una importante presencia en la tercera edición de Barcelona Games World, celebrado entre el 29 de noviembre al 2 de diciembre de 2018, con puestos de juego y un espacio donde podían degustarse sus hamburguesas. En el ámbito deportivo, en mayo de 2019 se anunció en Twitter una colaboración con la marca deportiva Nike Air Jordan que incluía nuevos escenarios en la actualización 9.1.0 y complementos para equipar a los personajes del mismo. En este sentido destaca también la Copa NeymarJR., celebrada en marzo de 2021, fruto de la colaboración con el futbolista brasileño y jugador del Paris Saint Germain. En junio de 2021 se pone en funcionamiento la sección «Ídolos» para agrupar eventos relacionados con deportistas internacionales como: Harry Kane (futbolista británico que milita en la selección inglesa y el club londinense Tottenham Hotspur), Marco Reus (destacado futbolista alemán del B. V. Borussia y la selección alemana de fútbol) o LeBron James (uno de los de los jugadores de la NBA más mediáticos). Por último, son destacables dos eventos asociados a marcas internacionalmente reconocidas como es el caso de Havaianas (la marca de calzado 
proveniente de Brasil) y Ferrari (una marca icónica que aprovechó la visibilidad del juego para promocionar su nuevo vehículo 256 GTB). Dentro de esta agrupación podemos destacar dos eventos que tratan de poner en valor determinadas habilidades o aptitudes personales como son: el «Concurso de dibujo magistral» de 2020 y el de «Fotografia furtiva» de 2021. En ambos, la participación requería de un inicio de sesión a través de cuentas en redes sociales y el registro mediante una cuenta de correo electrónico. Los premios consistieron en dinero del juego (paVos), juegos de mesa y objetos decorativos relacionados con el juego.

\subsection{INDUSTRIA MUSICAL}

La industria musical ha sido uno de los grandes recursos para la generación de eventos relevantes para los públicos del videojuego. En septiembre de 2018 tuvo lugar un evento entre el cantante canadiense Drake Graham (Drake) y el youtuber Ninja. A través de la plataforma TwicthTv ambos personajes jugaron una partida que fue retransmitida en streaming y que en la primera media hora ya acumulaba más de 600.000 espectadores online. En el transcurso del juego se incorporaron, además, otros personajes populares para el público estadounidense como el jugador de la NFL, JuJu Smith-Shuster; el músico de rap Travis Scott o Kim-Dotcom, fundador de Megaupload. El 2 de febrero de 2019 más de 10 millones de jugadores de Fortnite presenciaron dentro del propio juego, una actuación en directo del productor y DJ Marshmello. A principios de abril de 2020 la compañía anunciaba en Twitter un concierto dentro del juego del artista Travis Scott, donde presentaría un nuevo tema musical. En 2020 destacan las colaboraciones con artistas como el artista colombiano JBalvin que emitió un concierto en directo a través del juego y presentó una nueva canción dentro del evento denominado Fiesta Ultratumba, con el grupo de K-pop (Korean Pop) BTS que estrenaron un nuevo videoclip; los DJ's Kaskade; Diplo; Major Lazer; Noah Cyrus; el grupo de R\&B alternativo británico EasyLife; el cantante LILNasX, cuyo éxito vino precedido de su viral actividad en Twitter, o la cantante y actriz Ariana Grande, ganadora de un Grammy en 2020.

\subsection{Eventos estacionales y CUlturales}

En octubre de 2018 y con motivo de la actualización 6.20 del juego se generaron eventos relacionados con la fiesta de Halloween y el día de los muertos en México. El juego ofrecía vestimentas para los jugadores inspiradas en toreros y catrinas, así como guitarras y alebrijes tradicionales de México. Una costumbre arraigada por los desarrolladores del juego es hacer coincidir actualizaciones con momentos del año o festividades. Es el caso de la inclusión de personajes u objetos como el Cascanueces, Cascabella, la esfera de nieve en diciembre de 2018, el personaje de Cupido en febrero de 2019, coincidiendo con el día de San Valentín o el Festival de Invierno en la Na- 
vidad de ese mismo año. La festividad de San Valentín o las celebraciones de Pascua y primavera son usualmente puntos de referencia para generar eventos.

\subsection{Cruce con deportes tradicionales}

Con motivo del Australia Open 2019 y 2020 de tenis en Melbourne, se organiza la Fortnite Summer Smash. El evento consistió en una competición individual entre los mejores jugadores australianos. En 2020 el evento se desarrolló en paralelo a la competición real e incluyó además un torneo de exhibición dotado con premios de 500.000 y 400.000 dólares para destinar a causas benéficas. La Copa Uefa EURO 2020 de fútbol en junio y julio de 2021 fue uno de los primeros grandes eventos deportivos tras la pandemia. Este acontecimiento fue aprovechado por el videojuego para crear un evento especial. El director de marketing de la UEFA declaraba a este respecto: «Enormes audiencias estarán pegadas a la UEFA EURO 2020 este verano, por lo que parecía algo natural permitir que los millones de jugadores de Fortnite sean también parte de la acción» (UEFA, 2021). Los jugadores con mejores resultados en este evento pudieron ganar premios en metálico y recompensas en el juego. En junio de 2021 la NBA también llegó a Fortnite. Ante la celebración de los Playoffs finales de la competición por excelencia del baloncesto mundial, el evento Fortnite $X N B A$ se definió como un crossover para traer lo mejor del baloncesto a la tienda de objetos y al modo Creativo de Fortnite.

\subsection{Eventos propios o CON otros Videojuegos}

El 27 de agosto de 2019 llegó a Fortnite el evento de colaboración con el videojuego Borderlands un videojuego creado por Gearbox Software y 2KGames y ambientado en el space western y los cásicos de ciencia ficción. Para el final de la temporada 10 en octubre de 2019, Epic Games lanzó mensajes misteriosos en sus redes sociales (última parada; sin tiempo). Un meteorito había caído supuestamente en el juego y dejó tanto las redes sociales como el propio juego con pantallas en negro, fruto de un agujero negro que había absorbido todo. En febrero de 2020 se anunciaba a través del blog de la compañía el evento "Copa Celebración» exclusivo para usuarios de PlayStation 4, de dos días de duración y dotado de 1.000.000 de dólares en premios. Dentro de los eventos propios destacamos el realizado para promocionar la figura (ídolo) Bugha del que hablamos más adelante

\subsection{EvENTOS PRESENCIALES}

El evento presencial más importante que ha tenido lugar durante el periodo de estudio es la Fortnite World Cup 2019, presentada el 22 de febrero de 2019. 
La primera fase comenzó el 13 de abril con las partidas clasificatorias en diferentes países. En España el evento comenzó en mayo de 2019 con el anuncio de colaboración con El Rubius. El youtuber español cuenta con 37,7 millones de suscriptores en su cuenta de YouTube (elrubiusOMG), 12,2 millones de seguidores en Instagram (@elrubiuswtf) y más de dos millones de seguidores en la plataforma TwitchTv (/rubius). La acción consistió en un torneo previo (\#Rubius Trial) entre youtubers durante el mes de mayo. El 28 de este mismo mes el propio Doblas publicó en Twitter un adelanto enigmático de la campaña que obtuvo 56.140 «me gusta» y 6.210 retweets. Un día después El Rubius emitió un video explicando la mecánica promocional (https://bit.ly/2lJAEuR). Este video obtuvo 5.909 .468 visualizaciones y 26.224 comentarios. El concurso consistió en realizar un mapa del juego en el menor tiempo posible (speedrun). Los tres mejores jugadores ganaron 15.000 euros y un viaje a Nueva York para disputar la fase final del Fortnite World Cup 2019, del 26 al 28 de julio. Una vez concluido el concurso, Rubius publicó un video dando a conocer a los ganadores del reto (4.504.638 visualizaciones y 9.332 comentarios) (https://bit.ly/2lJAEuR). En Twitter (https://bit.ly/2lM7ICu) consiguió 11.092 «me gusta» y 924 retweets. La fase final de la competición tuvo lugar en el estadio Arthur Ashe de Nueva York. Según la consultora NewZoo (https://bit.ly/2kzrQHU), la fase de calificación generó un promedio semanal de 700.000 horas de audiencia en vivo a través de los canales de Fortnite en TwitchTv y YouTube. Las finales, generaron 5.6 millones de horas de audiencia en los canales oficiales del videojuego. En el canal de TwitchTv se registraron 4.5 millones de horas y compitieron más de 40 millones de jugadores. El ganador de la competición individual fue Kyle Bugha Giersdorf, de 16 años. Tras recibir el premio de 3.000.000 de dólares vio como su canal personal de TwitchTv incrementaba exponencialmente su audiencia a más de 220.000 horas entre el 29 de julio y el 4 de agosto. Días después fue entrevistado por Jimmy Fallon en Tonight Show el espacio televisivo de la cadena NBC (https://bit.ly/2YOqBXh). En diciembre de 2020 y por motivo de la pandemia por COVID-19, la propietaria del juego anunció la cancelación de eventos con público para 2021.

\section{Conclusiones y discusión}

En relación a las características funcionales y las dinámicas promocionales del videojuego (objetivo 1), Fortnite se distingue por una serie de particularidades que, en virtud de la bibliografía consultada y de los resultados de nuestro análisis, pueden resumirse en los siguientes puntos: a) Fácil accesibilidad. Los usuarios del videojuego no tienen que realizar un pago inicial que pudiera actuar como barrera. b) Desarrollo gráfico. Contiene un desarrollo gráfico atractivo en el que los jugadores han de enfrentarse a continuos retos para avanzar y obtener premios. c) Renovación continua. El juego propone una renovación continua y planificada de escenarios, retos y eventos. La llegada de nuevas temporadas para el juego o de nuevos eventos genera 
una expectación constante en los usuarios y publicity en los medios de comunicación especializados. d) Medios. Los canales de comunicación oficiales del juego contribuyen a crear notoriedad e interés entre los públicos, generando noticias sobre eventos próximos o futuros, actualizaciones y promociones.

A partir del análisis de 20 eventos generados entre septiembre de 2018 y agosto de 2021 (objetivo 2), estos actos podrían significar una herramienta estratégica que, a través de los medios sociales persigue dinamizar el consumo renovando periódicamente el interés hacia el juego. De esta forma el juego deja de ser un producto finito para convertirse en un producto flexible, no acotado y en continua renovación. En el caso de Fortnite los eventos están llamados a generar vínculos con sus públicos a partir de la incorporación de diferentes referentes culturales. A juzgar por las características de estas referencias o temáticas podemos deducir que, adolescentes y jóvenes son los segmentos de población prioritarios en este caso. Esta conclusión unida a la constatación de que los eventos se generan preferentemente en el entorno virtual (más aún en tiempos de pandemia), coincide con las aportaciones de Prensky (2001); Soengas Pérez et al. (2019) o del Instituto de la Juventud en España (Injuve, 2020) y sus informes anuales. Asistimos a un abandono progresivo de los medios clásicos y un auge del consumo de información en las redes sociales como referencia informativa para los jóvenes. Escuchar música/radio, ver películas/series o el uso activo e intensivo de las redes sociales, se han convertido en fundamentos del ocio de manera transversal y mayoritaria. La juventud en 2021 es nativa digital, lo que implica que han desarrollado gran familiaridad con los entornos online, los cuales utilizan para informase, estudiar, relacionarse, comprar y entretenerse. Se han constituido en referentes identitarios ya sea de forma individual o colectiva, concebidos para la socialización virtual. Fortnite recurre a referentes que hemos categorizado en torno a siete grupos: Producciones cinematográficas; Marcas de hostelería, ocio y deportes; Industria musical; Eventos estacionales; Cruce con deportes tradicionales y eventos relacionados con el propio videojuego (incluidos los de carácter presencial) o con otros videojuegos, también denominados «crossover». De este modo, en la estrategia de Fortnite para llegar a jóvenes y adolescentes (principalmente) a través de eventos, se ven implicadas algunas de las marcas globales (empresariales y personales) más importantes del mundo: Burger King; Nike; Adidas; LeBron James; JBalvin; TheGrefg; Havaianas; NBA; StarWars; Ferrari; NeymarJR, etc. Estas marcas encuentran en el territorio de los videojuegos un campo abonado para la difusión de sus estrategias y tácticas corporativas, fundamentalmente destinadas a públicos jóvenes y adolescentes.

Los eventos analizados generados mantienen algunos rasgos comunes con las definiciones de pseudoevento y evento 2.0. (objetivo 3). No son eventos espontáneos, sino que se planifican. Se planean principalmente para el propósito inmediato de ser informados y reproducidos, es decir, para obtener publicity. Su relevancia mediática es un aval y un fin en sí mismo. Habilitan espacios online para favorecer la notoriedad, la participación activa y la interacción sin olvidar la fuerza de la presencia- 
lidad (Fortnite World Cup 2019). Se dirigen a aforos ilimitados sin tener dependencia de los medios de comunicación tradicionales. En este sentido los pseudoeventos y eventos 2.0 generados por Fortnite, suponen un importante reto para las relaciones públicas. Como actos planificados o programados, podrían encarnar una nueva tendencia donde estaría adquiriendo aún más protagonismo el conocimiento de los públicos y el estudio de todos aquellos elementos que ayudarán a generar vínculos efectivos, en el marco del interés mutuo que toda actividad de relaciones públicas debe perseguir.

Todo ello con una estrategia de irradiación comunicativa en una estrategia transmedia (Scolari, 2020). En este sentido, se persigue una conexión de tipo emocional con los públicos relacionados con esas series, películas, músicos y el propio videojuego, ampliando las relaciones experienciales como estrategia de marca (Campillo Alhama y Martínez Sala, 2019). Eso le permite conectar con estos públicos no directamente relacionados con el videojuego y esforzar los lazos de afectividad, cercanía y empatía con aquellos usuarios ya establecidos a través de la experiencia del juego. Eso supone nuevas narraciones de Fortnite mediante la co-participación en otros eventos y la posibilidad de imbricar estrategias de comunicación multicanal y multitemático.

Así, los eventos organizados de manera estratégica y planificada persiguen que Fortnite mantenga una presencia permanente en el ecosistema comunicativo con acciones que establecen vínculos con los diferentes segmentos de sus públicos. Es, sin duda, un claro ejemplo de transmedia branding puesto que con esta estrategia los individuos co-participan, co-crean, se apropian y recirculan informaciones y experiencias, estableciendo una narrativa común, de tipo afectivo, que integra a todos esos canales comunicativos formando una interacción y experiencia alrededor del videojuego.

\section{Contribución específica y orden de autoría en el artículo}

El orden de firma se basa en los siguientes criterios: Conocimientos e interés por el fenómeno de estudio (relación con su línea de investigación principal); concepto e idea original del trabajo y tiempo de dedicación al mismo. Antonio Raúl Fernández Rincón ha asumido la conceptualización del artículo; la redacción del texto y su normalización a las directrices de la revista; el análisis de eventos; la consulta de bibliografia y fuentes seleccionadas, así como el envío a la revista y su resumen. Pedro Antonio Hellín ha participado en la definición y pertinencia del estudio; la construcción del marco teórico y metodología de análisis, la búsqueda de información en diversas fuentes y la revisión final. Antonio Castillo Esparcia ha colaborado en la orientación general del estudio sobre eventos y relaciones públicas; la definición de objetivos; ha realizado aportaciones al marco teórico y ha colaborado en la redacción de conclusiones, así como en la edición del texto. 


\section{Referencias bibliográficas}

AEVI (2018). El sector de los videojuegos en España: impacto económico y escenarios fiscales.

Recuperado de http://www.aevi.org.es/web/wp-content/uploads/2018/01/1801_AEVI_ EstudioEconomico.pdf

Aced, C. (2013). Relaciones públicas 2.0: Cómo gestionar la comunicación corporativa en el entorno digital. Barcelona: UOC.

Allen, J. (2008). Event planning: The ultimate guide to successful meetings, corporate events, fundraising galas, conferences, conventions, incentives and other special events. Ontario: John Wiley \& Sons.

Arceo Vacas, J. L. (1988). Fundamentos para la Teoría y la Técnica de las Relaciones Públicas. Barcelona: ESRP-PPU.

Aarsand, P. (2013). Children's digital gaming cultures. En D. Lemish (ed.), The Routledge international handbook of children, adolescents and media (pp.120-126). Londres: Routledge.

Argenti, P. (2003). Corporate Communications. Nueva York: Higher Education.

Bowdin, G., Allen, J., Mcdonell, I., O’Toole, W. y Harris, R. (2006). Events management (3rd Edition). Nueva York: Routledge.

Cabrera Cabrera, M. y Almansa Martínez, A. (2016). El director de relaciones públicas en las grandes empresas españolas. Revista Internacional de Relaciones Públicas, 6(11), 113-134. doi: http://dx.doi.org/10.5783/RIRP-11-2016-07-113-134

Campillo, C., Ramos, I. y Castelló, A. (2014). La gestión estratégica de la marca en los eventos empresariales 2.0. Revista Internacional de Investigación en Comunicación aDResearch ESIC, 1(10), 52-73. doi: 10.7263/ADRESIC.010.003

Campillo Alhama, C. y Martínez Sala, A. B. (2019). Events 2.0 in the transmedia branding strategy of World Cultural Heritage Sites. El profesional de la información, 28(5). doi: 10.3145/epi.2019.sep.09

Carter M., Moore, K., Horst, H., Mavoa, J. y Gaspart L. (2020). Situating the appeal of Fortnite within children's changing play cultures. Games and Culture, 1-19. doi: $10.1177 / 1555412020913771$

Castells, M. (2009). Comunicación y poder. Madrid: Alianza.

Castillo Esparcia, A. (2005). Comunicación organizacional: Teorías y estudios. Málaga: Clave Aynadamar.

Castillo Esparcia, A. y Almansa Martínez, A. (2005). Relaciones públicas y tecnología de comunicación. Análisis de los sitios de prensa virtuales. Comunicação Digital, 2(3), 132149. doi: 10.11606/issn.2238-2593.organicom.2005.138902

Chan, S. (13 de agosto de 2018). Fortnite coaching is growing among kids and parents. VentureBeat, Esports. Recuperado de https://venturebeat.com/2018/08/13/fortnitecoaching-is-growing-among-kids-and-parents/

Díaz De Salas, S., Mendoza Martínez, V. M. y Porras Morales, C. M. (2011). Una guía para la elaboración de estudios de caso. Razón y Palabra, 75. Recuperado de http://www. razonypalabra.org.mx/N/N75/varia_75/01_Diaz_V75.pdf 
Edwards, L. H. (2012). Transmedia storytelling, corporate synergy, and audience expression. Global Media Journal, 12(20), 1-12. Recuperado de http://www.globalmediajournal. com/archive/gmj-volume-11-issue-21-year-2012.html

Estanyol I Casals, E. (2012). Nuevas tendencias en organización de eventos. Comein. Revista de los Estudios de ciencias de la información, 8. Recuperado de https://comein.uoc.edu/ divulgacio/comein/es/numero08/articles/Article-Elisenda-Estanyol.html

Fernández Souto, A. B., Puentes Rivera, I. y Vázquez Gestal, M. (2019). La gestión comunicativa de las grandes empresas en España: estructura, recursos y principales retos de sus responsables. Comunicación y Sociedad, 32(1), 161-177. doi: 10.15581/003.32.1.161-177

Flanagan, A. (13 de junio de 2018). Marshmello wins Fortnite tourney, raises $\$ 1$ million for charity (thanks to a ninja) npr, Music News. Recuperado de https://www.npr. org/2018/06/13/619615123/marshmello-wins-fortnite-tourney-raises-1-million-forcharity-thanks-to-a-ninja

Getz, D. (2012). Event Studies. Nueva York: Routledge.

Goldblatt, J. (1990). Special events: the art and science of celebration. Nueva York: Van Nostrand Reinhold Company.

Gómez García, S. (2007). Videojuegos: El desafio de un nuevo medio a la comunicación social. Historia y Comunicación Social, 12, 71-82.

Injuve (2020). Informe Juventud en España. Recuperado de http://www.injuve.es/sites/ default/files/adjuntos/2021/03/informe_juventud_espana_2020.pdf

Libert, B. y Spector, J. (2008). We are smarter than me. New Jersey: Pearson Education.

Marlatt, R. (2019). Capitalizing on the craze of Fortnite: Toward a conceptual framework for understanding how gamers construct communities of practice. Journal of Education, 200(1), 3-11. doi:10.1177/0022057419864531

Matilla, K. y Marca Francés, G. (2011). La función estratégica del dircom en España en 2010. Revista Internacional de Relaciones Públicas, 1(2), 11-23. Recuperado de http:// revistarelacionespublicas.uma.es/index.php/revrrpp/article/view/19/32

Nicolás Ojeda, M. A., San Nicolás Romera, C. y Ros Velasco, J. (2019). In the Warcraft universe we trust: An analysis of transmedia advertising strategies in the World of Warcraft video game series (Battle Chest 3.0, Cataclysm, and Mists of Pandaria). International Journal of Communication, 3. doi: 1932-8036/20190005

Ollivier, B. (2010). Les ciéncies de la comunicació. Teories i aportacions. Bellaterra: UAB.

Palmeri, C. y Pendleton, D. (12 de agosto de 2018). Fortnite phenomenon turns «workaholic» game developer into billionaire. The Seattle Times. Recuperado de https://www. seattletimes.com/business/technology/fortnite-phenomenon-turns-workaholic-gamedeveloper-into-billionaire/

Parker, J. (16 de agosto de 2018). Fortnite: The complete guide to the biggest battle royale game. C/NET. Recuperado de http://real classicgames.com/guides/fortnite-thecomplete-guide-to-the-biggest-battle-royale-game/

Parkin, S. (27 de mayo de 2018). How Fortnite conquered the world. The Guardian. Recuperado de https://www.theguardian.com /games/2018/may/27/fortnite-conqueredthe-world-video-game-teenagers-parents 
Pedro Sebastiao, P. y Da Câmara Correia A. (2017). Eventos desportivos e a noticiabilidade. O caso da Lisboa Classic Regatta. Revista Internacional de Relaciones Públicas, 7(13), 141160. doi: 10.5783/RIRP-13-2017-09-141-160.

Prensky, M. (2001): Digital natives, digital immigrants. On the horizon, 9(5), 1-6.

Rodríguez, A. A. (2011). Eventos 2.0, el futuro ha llegado. Revista Protocolo. Actualidad, análisis y tendencias en la organización de eventos, 57, 62-65.

Rodríguez, A. A. (2014). La comunicación en vivo: eventos y visibilidad, clave en las organizaciones. Historia y Comunicación Social, 19, 167-179. doi: 10.5209/rev_HICS.2014. v19.44949

Rodríguez Gómez, G., Gil Flores, J. y García Jiménez, E. (1996). Metodología de la investigación cualitativa. Granada: Ediciones Aljibe.

San Nicolás Romera, C., Nicolás Ojeda, M. A. y Ros Velasco, J. (2015). Las narrativas publicitarias participativas de los videojuegos inspirados en el pasado histórico medieval: propuesta metodológica para su estudio en entornos comunicativos online. En C. San Nicolás Romera, y M. A. Nicolás Ojeda (coords.), Videojuegos y sociedad digital: nuevas realidades de estudio para la percepción del pasado histórico (pp. 111-127). Mar de Plata: Universidad Nacional Mar del Plata.

Scolari, C. A. (2009). Transmedia storytelling: implicit consumers, narrative worlds and branding in contemporary media production. International Journal of Communication, 4(3), 586-606. Recuperado de https://ijoc.org/index.php/ijoc/article/view/477/336

Scolari, C. A. (2020). Transmedia, literacy and participatory culture. A research agenda. En J. C. Mateu., P. Andrada y T. Quiroz, Media Education in Latin America. (pp. 248-258). Londres: Routledge.

Scolari, C. A., Lugo Rodríguez, N. y Masanet, M. J. (2019). Transmedia education. From the contents generated by the users to the contents generated by the students. Revista Latina de Comunicación Social, 74, 116-132. doi: 10.4185/RLCS-2018-1324en

Seo, Y. (2016). Professionalized consumption and identity transformations in the field of eSports. Journal of Business Research, 69, 264-272.

Soengas Pérez, X., López Cepeda, A. M. y Sixto García, J. (2019). Dieta mediática, hábitos de consumo de noticias y desinformación en los universitarios españoles. Revista Latina de Comunicación Social, 74, 1056-1070. doi: 10.4185/RLCS-2019-1371-54

Tassi, T. (13 de agosto de 2018). Fortnite: Battle royale season 5, week 6 challenges leak online. Forbes Games. Recuperado de https://www.forbes.com/sites/ insertcoin/2018/08/13 /fortnite-battle-royale-season-5-week-6-challenges-leak-online/\#50fddd973dc9 (12-08-2019).

UEFA (7 de junio de 2021). La UEFA EURO 2020, presente en Fortnite. Recuperado de https://es.uefa.com/insideuefa/about-uefa/news/026a-12769dc898de-e3698bef49571000--la-uefa-euro-2020-presente-en-fortnite/

Xifra, J. (2007). Técnicas de relaciones públicas. Barcelona: Editorial UOC.

Xifra, J. (2010). Relaciones públicas, empresa y sociedad: una aproximación ética. Barcelona: Editorial UOC. 
Xifra, J. (2012). Manual de relaciones públicas e institucionales. Madrid: Tecnos.

Yeoman, I., Robertson, M., Ali Knight, J., Drummond, S. y Mcmahon Beattie, U. (2004). Festival and events management. An International Arts and Culture Perspective. Oxford: Elsevier Butterworth-Heinemann.

Yin, R. K. (1994). Discovering the future of the case study method in evaluation research. Evaluation Practice, 15(3), 283-290. 